\title{
Una visión de alumnos universitarios sobre la relación de sus padres como pareja
}

Patricia Ortega*, Patricia Plancarte, Adriana Garrido, Adriana Reyes y Laura Torres.

Facultad de Estudios Profesionales Iztacala, Universidad Nacional Autónoma de México, México.

Recibido, agosto 24/2017

Concepto de evaluación, septiembre 21/2017

Aceptado, noviembre 23/2017
Referencia: Ortega, P., Plancarte, P., Garrido, A., Reyes, A. \& Torres, L. (2018). Una visión de alumnos universitarios: relación de padres como pareja. Acta colombiana de Psicología, 21(2), 88-98. doi: http:// www.dx.doi.org/10.14718/ACP.2018.21.2.5

Resumen

En una relación de pareja, sus integrantes comparten expectativas relacionadas con lo que vivieron y aprendieron en su familia de origen, de manera que la nueva familia reflejará patrones que los hijos reproducirán en un futuro. Si los hijos perciben relaciones de pareja adecuadas, retomarán estas mismas relaciones en la formación de su nueva familia; sin embargo, cuando esta relación se ve afectada por la separación, es probable que existan diferencias importantes en la percepción de los hijos. El objetivo del presente artículo fue analizar la percepción de universitarios respecto a la relación de sus padres como pareja dentro de la dinámica familiar, tomando en cuenta si sus padres vivían juntos o separados. Se utilizó una muestra intencional compuesta por 566 alumnos universitarios, de los cuales 345 eran mujeres y 221, hombres. Se aplicó el cuestionario Dinámica de la Familia Nuclear Mexicana de Vásquez et al. (2003), aunque solo se abordó el área "Los padres. Su dinámica cómo pareja". La diferencia estadística entre grupos se obtuvo a través de la prueba U de Mann-Whitney. Los resultados mostraron diferencias estadísticamente significativas entre la percepción de los universitarios con respecto a sus padres cuando viven juntos o cuando viven separados. Se concluyó que existen diferentes factores que afectan el funcionamiento de la dinámica familiar, los cuales influyen de manera diferencial en la percepción que puede tener cada miembro de la familia.

Palabras clave: universitarios, padres, relación de pareja.

\section{Perception of university students about their parents' relationship as a couple}

\begin{abstract}
When a couple starts a new family, both members share expectations based on what they have learned from their experience in their respective families of origin, in such a way that their offspring will repeat the same patterns in the future. If children perceive a healthy appropriate relationship between their parents, chances are that they too will have a good relationship when they start their own families. However, children will have a different perception when their parents go through a separation or divorce. Based on these facts, the aim of this article was to analyze university students' perception regarding their parents' relationship as a couple within their family environment, taking into consideration whether their parents lived together or separately. A target group comprised of 566 university students of which 345 were women and 221 were men participated in the study. The instrument used was the measurement questionnaire "Dinámica de la Familia Nuclear Mexicana" [The Mexican Family Dynamics] (Vásquez, et al., 2003). In this study the only area addressed was "Parents and their relationship as a couple". The statistical difference between groups was obtained using the Mann-Whitney U test. The results showed significant statistical differences between university students' perception of their parents depending on whether they live together or separately. A conclusion was reached on the existence of different factors that affect the family environment, which has a differential influence on each family member's perception.

Key words: University students, parents, relationship as a couple.
\end{abstract}

* Av. Barrios 1, Reyes Iztacala, Tlalnepantla, Edo. México. Tel.+525558871561, patosi75@gmail.com 


\title{
Uma visáo de alunos universitários sobre a relaçáo de seus pais como casal
}

\author{
Resumo
}

\begin{abstract}
Em uma relação de casal, seus integrantes compartilham expectativas relacionadas com o que viveram e aprenderam em sua família de origem, de forma que a nova família refletirá padrões que os filhos reproduzirão no futuro. Se os filhos percebem relações de casal adequadas, retomarão essas mesmas relações na formação da sua nova família; no entanto, quando essa relação se vê afetada pela separação, é provável que existam diferenças importantes na percepção dos filhos. O objetivo do presente artigo foi analisar a percepção de universitários com respeito à relação de seus pais como casal dentro da dinâmica familiar, levando em consideração se seus pais viviam juntos ou separados. Utilizou-se uma amostra intencional composta por 566 alunos universitários, dos quais 345 eram mulheres e 221, homens. Aplicou-se o questionário Dinâmica da Família Nuclear Mexicana, de Vásquez et al. (2003), ainda que apenas a área "Os pais - sua dinâmica como casal" tenha sido abordada. A diferença estatística entre grupos foi obtida através do Teste U de Mann-Whitney. Os resultados mostraram diferenças estatisticamente significativas entre a percepção dos universitários com respeito a seus pais quando vivem juntos ou quando vivem separados. Concluiu-se que existem diferentes fatores que afetam o funcionamento da dinâmica familiar, os quais influenciam de maneira diferencial na percepção que cada membro da família pode ter.

Palavras-chave: pais, relação de casal, universitários.
\end{abstract}

\section{INTRODUCCIÓN}

La necesidad de todo ser humano de vivir en familia se pondera tomando en cuenta el aspecto psicológico que tiene la relación niño-adulto durante el proceso de desarrollo en el cual crece. Las personas adultas (padre y madre) y los hermanos que conviven con el niño son generalmente quienes constituyen la familia, y a partir de la convivencia entre los miembros se dan ciertos procesos psicológicos que determinarán el desarrollo de la personalidad de los hijos, la socialización, la autonomía, las relaciones interpersonales, y su preparación para las relaciones de pareja y para la vida familiar en general. Al respecto, Padilla y Díaz-Loving (2013) señalan que la familia es el ámbito privilegiado de socialización en donde se moldea el carácter de los individuos y se promueven modos de actuar y de pensar que a la larga se convertirán en hábitos; por lo que la familia también funciona como espacio productor y transmisor de patrones y prácticas culturales.

La familia también es definida como una unidad que crece y se desarrolla como algo dinámico, evolutivo, y con normas de disciplina, escalas de valores y actitudes que permiten definir los estilos de vida y dinámicas de relación entre sus integrantes (Torres, Reyes, Ortega \& Garrido, 2015). Es por esto que Estrada (1993) señala dos grandes objetivos de toda familia: (1) resolver las tareas o crisis que enfrenta la familia en las diferentes etapas de desarrollo y (2) aportar los complementos a las necesidades de sus miembros y lograr una satisfacción en el presente, así como una preparación segura y adecuada para el futuro.

La función de la familia va más allá de garantizar la supervivencia y el crecimiento físico, ya que implica la promoción del desarrollo social y afectivo de los hijos que repercutirá hasta la adultez; y es por esto que las relaciones que se presenten en la familia tendrán una consecuencia en dicho desarrollo (Vallejo, Sánchez-Barranco \& SánchezBarranco, 2004). Además, algunos autores consideran que existe una relación bidireccional entre padres e hijos, es decir, que los hijos ya no fungen como espectadores pasivos, sino que intervienen en la dinámica de la interacción familiar y en las relaciones con sus padres (Oudhof \& Robles, 2014).

Por otra parte, la dinámica familiar surge como un conjunto de relaciones de cooperación, intercambio, poder y conflicto que se establecen en el interior de las familias (Torres, Ortega, Garrido \& Reyes, 2008), y es en la pareja donde se representa la parte central o medular de la familia nuclear, y donde se crean expectativas acerca de cómo se lleva a cabo la vida futura en pareja, contemplando el tener hijos e hijas, cuidarlos, atenderlos y lograr una armonía familiar (Garrido, Reyes, Torres \& Ortega, 2008).

Ahora bien, es relevante señalar que una relación de pareja es una parte importante en la vida de las personas, ya que en ella se muestran sentimientos de afecto que le permiten a cada persona lograr una identidad personal que satisfaga sus necesidades de seguridad, confianza y afecto, así como las de orden sexual (García, 2010; Silva \& Ortiz, 2014; Soto, 2015). Es importante considerar que, al formar una pareja, sus integrantes comparten expectativas relacionadas con lo que vivieron y aprendieron en su familia de origen (Garrido et al. 2008; Padilla \& Díaz-Loving, 2013); y, asimismo, la nueva familia reflejará patrones que sus hijos reproducirán en un futuro, de manera que si ellos perciben relaciones de pareja adecuadas en sus padres, retomarán estas mismas estrategias en la formación de su 
nueva familia -como al integrar una buena comunicación, compartir metas y aspiraciones, tener concordancia de ideas, expresar manifestaciones amorosas y tener compatibilidad de caracteres, sin olvidar el buen manejo de emociones como el odio y las actitudes de agresión-; sin embargo, si perciben relaciones conflictivas, dichos patrones pueden influir de manera negativa en la formación de su nueva familia.

Aunque formar una nueva pareja es algo relativamente fácil, vivir las exigencias y responsabilidades que esto implica no lo es, ya que es necesario identificar pautas de interacción favorables entre la pareja que, si no se logran, pueden presentar un conflicto entre las expectativas deseadas y las reales que puede llevar a una relación conflictiva que va a interferir con el desarrollo de la futura familia (Padilla \& Díaz-Loving, 2013). Específicamente, los acontecimientos en las relaciones de pareja son diferentes para cada una de ellas, y, en ocasiones, cuando se identifican periodos de crisis, se atraviesa por distintas modificaciones estructurales y funcionales que afectan la estabilidad de la pareja y requieren de un reajuste al interior de cada familia, lo que en muchos casos lleva a la separación de los padres y a la modificación de las relaciones que se dan entre cada uno de los miembros de la familia, principalmente entre las relaciones de pareja.

En muchos casos, la desarmonía en la pareja lleva a que los padres consideren vivir de manera separada, ruptura que puede deberse, sobre todo, a malos tratos físicos o psicológicos, a la incompatibilidad de caracteres, a la ponderación de intereses económicos, personales y sociales, al incumplimiento de deberes respecto a los hijos, o a problemas de adicciones, entre otros; aspectos que facilitan el surgimiento de presiones que llevan a un estrés marital y a una fragmentación familiar (García, Rivera-Aragón, Diaz-Loving \& Reyes-Lagunes 2015; Lemes, 2016).

En este sentido, los padres tienen una influencia decisiva en el desarrollo de sus hijos, y la separación conlleva a una etapa en la cual todo el contexto familiar cambia -principalmente, las relaciones de la pareja- y a la pérdida del equilibrio y la aparición de conflictos tanto para los padres como para los hijos (De la Torre, 2005; Fontana \& Fernández, 2011; Lemes, 2016; Valdés \& Aguilar, 2012). Partiendo de esto, la forma en que los padres tratan los conflictos antes y después de la separación puede influir en la manera de relacionarse con sus hijos, así como en la interacción entre ellos mismos, por lo que algunos autores recomiendan no pasar desapercibida dicha dinámica familiar y evaluarla con el fin de identificar y proponer las mejores estrategias de interacción que lleven a tener contactos adecuados en la relación con sus hijos (Arch, 2010; O’Donohue, Beitz \& Cummings, 2008).
En el caso de los jóvenes, los estudios han señalado que la experiencia de vivir con sus padres juntos o separados les ha permitido tener conciencia de las posibles dificultades a las que se pueden enfrentar; y en los casos donde los jóvenes aún no han establecido un vínculo matrimonial, estos expresan la convicción de que serán más cuidadosos y serios al momento de consolidar una pareja, además de que no repetirán el modelo de relación de sus padres y madres (Durán, Medina, González \& Rolón, 2007).

Asimismo, la literatura demuestra que los jóvenes o estudiantes universitarios con padres separados, aun cuando funcionan bien, muestran sentimientos dolorosos, creencias y recuerdos, así como preocupaciones por la separación de sus padres -aunque algunos lo aceptan y no presentan indicadores clínicos-, y se ha evidenciado que aquellos que viven en familias separadas tienen más problemas en sus relaciones familiares que los estudiantes con familias intactas; que las tensiones y preocupaciones están relacionadas con temas como la decisión de con quién vivir, la frecuencia de contacto con el padre que se va y el conflicto entre los padres; que el contacto con el padre o madre ausente es importante para los hijos de las familias separadas; y que el malestar que provoca la separación no se atribuye a la situación misma, sino a las tensiones causadas por el conflicto parental (Durán et al., 2007). Incluso, se ha considerado que la ausencia de los padres puede ser un factor de riesgo que afecta la percepción de los hijos en cuanto a su propio matrimonio, además de que aumenta la posibilidad de repetir la situación de separación, visión que ocasiona menor optimismo con respecto al éxito de su futuro matrimonio (Durán et al., 2007).

Por otra parte, algunas investigaciones (Durán et al., 2007; García, 2010; García \& Guzmán, 2010; Silva \& Ortiz, 2014; Soto, 2015) han analizado las características personales principales en la elección de pareja por parte de los jóvenes, $\mathrm{y}$ al respecto señalan que las mujeres esperan construir una relación caracterizada por la estabilidad, la seriedad, el complemento mutuo, la madurez y tener un nivel de educación similar a su pareja, en la cual se brinde compañía, inteligencia, madurez emocional, respeto, sinceridad y fidelidad; mientras que los hombres, por su parte, esperan encontrar en su pareja honestidad, respeto, un físico agradable, inteligencia, flexibilidad y entendimiento en el aspecto sexual y castidad.

En la actualidad, los jóvenes no ven el matrimonio o la elección de pareja como un compromiso institucional, sino como una forma de compañerismo en donde existe una posibilidad más abierta de escoger a la pareja y encontrar el amor, la confianza y el cariño, siendo estos los valores contemporáneos a la hora de elegir pareja y formar una familia (García, 2010). Sin embargo, la elección de pareja 
en tiempos pasados estuvo marcada por la imposición, ya sea de los padres, la comunidad, los jefes o la familia, y aunque actualmente dicha elección es libre y responde a una elección personal (García, 2010; García \& Martínez, 2010), los patrones familiares pueden influir en la conformación de las futuras parejas de los hijos.

Teniendo esto en cuenta, el objetivo del presente trabajo fue analizar la percepción de universitarios con respecto a la formación de sus padres como pareja dentro de la dinámica familiar, considerando si estos vivían juntos o separados.

\section{MÉTODO}

\section{Tipo de estudio}

Se llevó a cabo un estudio comparativo para analizar la relación entre las variables a partir de las diferencias existentes entre dos o más grupos de individuos de acuerdo con sus respectivas características. Se trata de un diseño no experimental, ya que no utiliza variables manipuladas, y los participantes no son asignados aleatoriamente (Ato \& Vallejo, 2015).

\section{Participantes}

La muestra estuvo compuesta por 566 alumnos universitarios solteros de la ciudad de México con edades entre los 18 y 22 años $(M=20.58 ; \mathrm{DE}=2.97)$, de los cuales 345 eran mujeres y 221 , hombres. Todos cursaban la carrera de Psicología en una de las Facultades de la Universidad Nacional Autónoma de México, en los semestres de primero a séptimo. El $68 \%$ vivía con sus padres y el resto (32\%) en familias separadas.

Los estudiantes fueron elegidos de manera intencional mediante un muestreo no probabilístico. Su participación fue voluntaria, tras firmar un consentimiento informado en el que se describían los objetivos del estudio, la institución que lo avala, la confidencialidad de la información recopilada y los datos generales de los investigadores.

\section{Instrumento}

Se aplicó el cuestionario "Dinámica de la Familia Nuclear Mexicana”, elaborado y validado por Vásquez et al. (2003). Este instrumento consta de 160 preguntas y ocho áreas de comportamiento: (1) "Los padres. Su dinámica como pareja", (2) "Formación de identidad", (3) "Comunicación, expresión, solidaridad", (4) "Estructura de autoridad", (5) "Manejo del conflicto y agresividad", (6) "Disciplina y método", (7) "Sistema de valores", y (8) "Aislamiento e integración sociocultural".
El cuestionario cuenta con validación en diferentes estudios y poblaciones mexicanas, en donde se ha demostrado una consistencia interna significativa con un valor del alfa de Cronbach de .941 (Vásquez et al., 2003). Específicamente, su estilo de respuesta es de dos opciones $(\mathrm{Sí}=1$ y No $=0$ ) para cada una de las preguntas. Para este trabajo, el rango de respuestas fue de 0 a 36, donde el valor más alto indicaba una mejor dinámica familiar. Los valores se presentan en términos de porcentajes y para la comparación entre poblaciones se utilizan las puntuaciones medias.

Los participantes respondieron el total del cuestionario, pero en este trabajo solo se analizó el área correspondiente a "Los padres. Su dinámica como pareja" para dar cumplimiento al objetivo propuesto. En esta área de comportamiento se considera a la pareja como la parte central de la familia nuclear que conlleva a un proceso de unidad familiar: padre, madre e hijos. Finalmente, se hizo una evaluación tomando en cuenta seis indicadores: (a) aspiraciones y metas comunes, (b) odio-agresión, (c) concordancia de ideología, (d) amor, (e) compatibilidad de caracteres y (f) comunicación.

\section{Procedimiento}

La aplicación del cuestionario se llevó a cabo en las instalaciones de la universidad a la que asistían los jóvenes. En primera instancia, se les informó el objetivo de la investigación, se les solicitó su cooperación voluntaria, y a quienes aceptaron responder el cuestionario se les pidió firmar un consentimiento informado. A continuación, se respondieron las preguntas que surgieron sobre la investigación, se enfatizó sobre su propósito e importancia en el ámbito científico, y se les aseguró la confidencialidad de la información que se obtendría. Una vez aclaradas las dudas y comentarios del estudio se hizo entrega de los cuestionarios y se les solicitó que completaran la información relacionada con los datos generales. Posteriormente, se leyó junto a ellos la siguiente instrucción: "Lee con detenimiento las siguientes preguntas y marca la opción que más refleje tu dinámica familiar, recuerda que no hay respuestas correctas ni incorrectas y que la información será utilizada de manera confidencial para la investigación".

Los participantes debían responder afirmativa o negativamente a cada pregunta, de acuerdo con lo que representaba mejor la dinámica dentro de su familia, sobre todo, con respecto a su padre o su madre biológicos. Por último, se informó que no tenían un tiempo límite para responderlo y que trataran de ser lo más veraces posible. La duración aproximada para responder el cuestionario fue de 30 minutos.

Al concluir, se recopilaron los cuestionarios, se agradeció la participación y, posteriormente, se realizó la captura de la 
información en el programa estadístico SPSS con el propósito de llevar a cabo el análisis relacionado con el área a estudiar.

\section{Análisis de datos}

Los resultados se analizaron por medio del paquete estadístico SPSS, donde se consideró la percepción que tienen los universitarios sobre la dinámica como pareja de sus padres. Para ello, se tomaron en cuenta seis indicadores en relación con el padre y la madre: (a) aspiraciones y metas comunes, (b) odio-agresión, (c) concordancia de ideología, (d) amor, (e) compatibilidad de caracteres y (f) comunicación.

Para comparar la percepción de los universitarios en relación con la condición familiar en la que viven se realizó la prueba de normalidad Kolmogorov-Smirnov, donde se encontró una asimetría negativa en la distribución, lo que indica que la muestra no corresponde a una distribución normal, y por lo que se realizaron las comparaciones a través de la prueba de Wilcoxon Mann Whitney o U de Mann Whitney.

\section{RESULTADOS}

Con el propósito de identificar el contenido de cada indicador, a continuación se especifican las preguntas que formaron parte de cada uno de los indicadores de la investigación (véase Tabla 1).

Tabla 1

Preguntas de cada uno de los indicadores implicados en el área de comportamiento "Los padres. Su dinámica como pareja"

\begin{tabular}{|c|c|c|}
\hline Indicador & Pregunta & Total \\
\hline \multirow{6}{*}{$\begin{array}{l}\text { 1. Aspiraciones y } \\
\text { metas comunes }\end{array}$} & 30. ¿El manejo del ingreso familiar es compartido por tu papá con su pareja? & \multirow{6}{*}{6} \\
\hline & 31. ¿El manejo del ingreso familiar es compartido por tu mamá con su pareja? & \\
\hline & 76. ¿Tu papá cree que su unión es para toda la vida? & \\
\hline & 77. ¿Tu mamá cree que su unión es para toda la vida? & \\
\hline & 131. ¿Comparte tu papá, con su mamá sus metas, aspiraciones y proyectos? & \\
\hline & 132. ¿Comparte tu mamá, con tu papá sus metas, aspiraciones y proyectos? & \\
\hline \multirow{6}{*}{$\begin{array}{l}\text { 2. Odio agresión } \\
\text { (física y psicológica) }\end{array}$} & 26. ¿Tu papá ha agredido físicamente a su pareja? & \multirow{6}{*}{6} \\
\hline & 27. ¿Tu mamá ha agredido físicamente a su pareja? & \\
\hline & 46. ¿Tu papá piensa con frecuencia que su pareja la odia? & \\
\hline & 47. ¿Tu mamá piensa con frecuencia que su pareja la odia? & \\
\hline & 48. ¿Tu papá ha golpeado a su pareja? & \\
\hline & 49. ¿Tu mamá ha golpeado a su pareja? & \\
\hline \multirow{7}{*}{$\begin{array}{l}\text { 3. Concordancia de } \\
\text { ideología }\end{array}$} & 13. ¿Tu papá, crítica con frecuencia la forma de ser de su pareja? & \multirow{7}{*}{7} \\
\hline & 14. ¿Tu mamá crítica con frecuencia la forma de ser de su pareja? & \\
\hline & 33. ¿Considera tu papá que tu mamá es un ejemplo para seguir? & \\
\hline & 34. ¿Considera tu mamá que tu papá es un ejemplo para seguir? & \\
\hline & 54. ¿Tus padres tienen formas de pensar muy parecidas? & \\
\hline & 88. ¿Tu papá depende totalmente de tu mamá? & \\
\hline & 89. ¿Tu mamá depende totalmente de tu papá? & \\
\hline \multirow{6}{*}{ 4. Amor } & 73. ¿Tu papá admira a tu mamá? & \multirow{6}{*}{6} \\
\hline & 74. ¿Tu mamá admira a tu papá? & \\
\hline & 83. ¿Piensa tu papá que tu mamá lo quiere? & \\
\hline & 84. ¿Piensa tu mamá que tu papá la quiere? & \\
\hline & 126. ¿Tu papá se siente separado sentimentalmente de su pareja, aunque viven juntos? & \\
\hline & 127. ¿Tu mamá se siente separada sentimentalmente de su pareja, aunque viven juntos? & \\
\hline
\end{tabular}




\begin{tabular}{ll}
\hline \multicolumn{1}{c}{ Indicador } & \multicolumn{1}{c}{ Pregunta } \\
\hline & 40. ¿A tu papá generalmente le disgusta el comportamiento de su pareja? \\
& 41. ¿A tu mamá generalmente le disgusta el comportamiento de su pareja? \\
& 50. ¿Tu mamá y tu papá discuten con mucha frecuencia? \\
& 69. ¿Tu papá ha abandonado por más de 24 horas la casa a consecuencia de un dis- \\
5. Compatibilidad de & gusto \\
caracteres & g0. ¿Tu mamá ha abandonado por más de 24 horas la casa a consecuencia de un dis- \\
& gusto? \\
& 90. ¿Cuándo discuten frecuentemente alguno pierde por completo el control? \\
& 55. ¿La mayoría de las decisiones en casa las toman tu papá considerando a tu mamá? \\
& 56. ¿La mayoría de las decisiones en casa las toman tu mamá considerando a tu papá? \\
& 61. ¿Los momentos difíciles suelen resolverlos juntos? \\
& 108. ¿Cree tu papá que después de los años de vivir juntos conoce a tu mamá? \\
& 109. ¿Cree tu mamá que después de los años de vivir juntos conoce a tu papá?
\end{tabular}

En la Figura 1 se presenta el porcentaje de respuestas afirmativas por parte de los estudiantes en cada uno de los seis indicadores, considerando si sus familias vivían juntas o separadas. Los datos muestran que los indicadores con mayor porcentaje en las familias que están juntas $(80 \%)$ fueron los de aspiraciones, metas comunes y comunicación. Mientras que, cuando las familias se encuentran separadas, el porcentaje en el indicador de aspiraciones es menor (23.63\%) y en el de comunicación alcanza un 39.26\%.

Por otra parte, el porcentaje en el indicador de odio y agresión cuando las familias viven juntas fue de $11.2 \%$, mientras que cuando se encuentran separadas fue mayor (27.68 \%); el porcentaje para el indicador de compatibilidad de caracteres cuando las familias viven juntas fue menor (26.01\%), mientras que al estar separadas fue de $42.42 \%$; y en los indicadores de concordancia de ideologías y amor, los resultados reflejan porcentajes similares tanto en familias que viven juntas como en familias separadas.

Con la intención de identificar si existían diferencias significativas entre las dos poblaciones, se realizó el análisis estadístico empleando la prueba U de Mann-Whitney para muestras independientes en cada uno de los indicadores estudiados. Y, como se puede apreciar en la Tabla 2, en todos los casos se observa la existencia de diferencias estadísticamente significativas entre la percepción de los universitarios con respecto a la relación de pareja de sus padres cuando viven juntos o separados.

Con el fin de identificar en qué aspectos de cada uno de los indicadores se encontraban las diferencias que percibían los universitarios acerca de la formación de los padres como pareja, a continuación se presentan las tablas 3, 4, 5, 6, 7 y 8 , en las que se muestra el porcentaje de respuestas a cada pregunta, dependiendo de cada indicador.

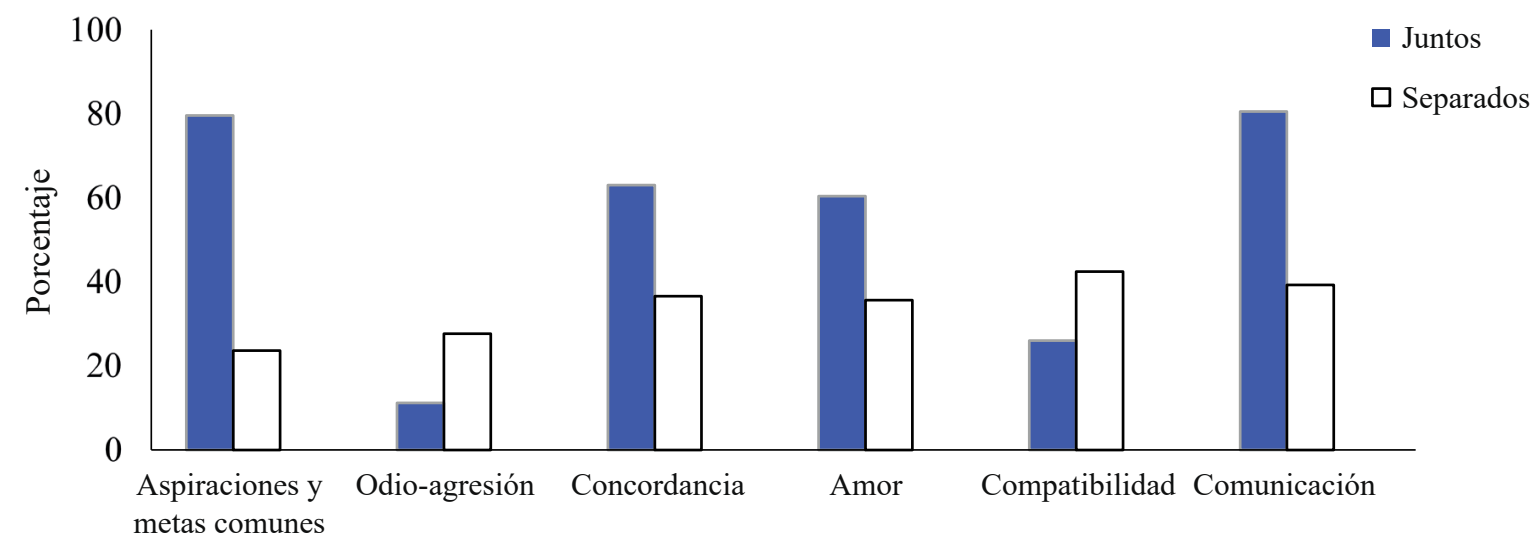

Figura 1. Dimensiones en la dinámica como pareja en las familias de universitarios. Muestra los porcentajes de respuestas afirmativas en cada uno de los indicadores de la dinámica como pareja cuando las familias viven juntas o separadas. 
Tabla 2

Resultados del análisis estadístico (U de Mann-Whitney) de acuerdo con ser padres o madres y la condición de convivencia familiar (juntos o separados)

\begin{tabular}{|c|c|c|c|c|}
\hline Madres/Padres & Convivencia familiar & Media & $\mathrm{U}$ & Sig. \\
\hline \multirow{2}{*}{ Madres. Aspiraciones } & Juntos & 2.3874 & \multirow{2}{*}{6646.00} & \multirow{2}{*}{.05} \\
\hline & Separados & .7273 & & \\
\hline \multirow{2}{*}{ Padres. Aspiraciones } & Juntos & 2.3874 & \multirow{2}{*}{6791.00} & \multirow{2}{*}{.05} \\
\hline & Separados & .6909 & & \\
\hline \multirow{2}{*}{ Madres. Odio agresión } & Juntos & 2.7277 & \multirow{2}{*}{27077.50} & \multirow{2}{*}{.05} \\
\hline & Separados & 2.5030 & & \\
\hline \multirow{2}{*}{ Padres. Odio agresión } & Juntos & 2.5995 & \multirow{2}{*}{20591.50} & \multirow{2}{*}{.05} \\
\hline & Separados & 1.8364 & & \\
\hline \multirow{2}{*}{ Madres. Ideologías } & Juntos & 2.1414 & \multirow{2}{*}{24369.50} & \multirow{2}{*}{.05} \\
\hline & Separados & 1.7879 & & \\
\hline \multirow{2}{*}{ Padres. Ideologías } & Juntos & 2.3639 & \multirow{2}{*}{21071.50} & \multirow{2}{*}{.05} \\
\hline & Separados & 1.8182 & & \\
\hline \multirow{2}{*}{ Madres. Amor } & Juntos & 2.2435 & \multirow{2}{*}{13953.00} & \multirow{2}{*}{.05} \\
\hline & Separados & 1.0364 & & \\
\hline \multirow{2}{*}{ Padres. Amor } & Juntos & 2.3377 & \multirow{2}{*}{13000.00} & \multirow{2}{*}{.05} \\
\hline & Separados & 1.1030 & & \\
\hline \multirow{2}{*}{$\begin{array}{l}\text { Madres. Compatibilidad } \\
\text { de caracteres }\end{array}$} & Juntos & 1.5079 & \multirow{2}{*}{27847.00} & \multirow{2}{*}{.05} \\
\hline & Separados & 1.3455 & & \\
\hline \multirow{2}{*}{$\begin{array}{l}\text { Padres. Compatibilidad } \\
\text { de caracteres }\end{array}$} & Juntos & 1.5314 & \multirow{2}{*}{17332.00} & \multirow{2}{*}{.05} \\
\hline & Separados & .8485 & & \\
\hline \multirow{2}{*}{ Madres. Comunicación } & Juntos & 1.6335 & \multirow{2}{*}{10895.00} & \multirow{2}{*}{.05} \\
\hline & Separados & .6121 & & \\
\hline \multirow{2}{*}{ Padres. Comunicación } & Juntos & 1.5471 & 1153300 & 05 \\
\hline & Separados & .5515 & 11533.00 & .05 \\
\hline
\end{tabular}

La Tabla 3 representa los porcentajes obtenidos en el indicador "aspiraciones y metas comunes". Específicamente, se observa la existencia de diferencias en la percepción de los universitarios en relación con la condición familiar en la que viven, de manera que cuando se encuentran las familias juntas, los porcentajes son más altos; es decir, que los universitarios consideran que cuando sus padres viven separados no comparten aspiraciones o metas comunes, donde se encuentra una diferencia significativa entre los grupos $\left(\mathrm{X}^{2}=293.07, \mathrm{p}<.05\right)$.

Con respecto al indicador "odio y agresión", los mayores porcentajes tanto para las familias que viven juntas como las separadas están asociados con el padre (preguntas 26, 46 y 48), es decir, que los estudiantes identifican mayor agresión en el padre que en la madre. Situación que se ratifica cuando las familias están separadas (véase Tabla 4).

Tabla 3

Porcentaje de respuestas afirmativas del indicador "aspiraciones y metas comunes" en familias que viven juntas y en familias separadas

\begin{tabular}{lcc}
\multicolumn{1}{c}{ Pregunta } & Juntos & Separados \\
\hline 30. ¿El manejo del ingreso familiar es compartido por tu papá con su pareja? & 88.7 & 29.7 \\
31. ¿El manejo del ingreso familiar es compartido por tu mamá con su pareja? & 85.6 & 31.5 \\
76. ¿Tu papá cree que su unión es para toda la vida? & 79.1 & 17 \\
77. ¿Tu mamá cree que su unión es para toda la vida? & 78 & 20.6 \\
131. ¿Comparte tu papá, con su mamá sus metas, aspiraciones y proyectos? & 70.9 & 22.4 \\
132. ¿Comparte tu mamá, con tu papá sus metas, aspiraciones y proyectos? & 75.1 & 20.6 \\
\hline Total & $79.56 \%$ & $23.63 \%$ \\
\hline
\end{tabular}

Nota. $\mathrm{X}^{2}=293.07, \mathrm{p}<.05$. 
En el análisis estadístico se encuentra que la diferencia es significativa entre los grupos $\left(\mathrm{X}^{2}=58.79, \mathrm{p}<.05\right)$.

Por otra parte, la pregunta que mejor ejemplifica el indicador "concordancia de ideologías" es la 54, "¿Tus padres tienen formas de pensar muy parecidas?", en donde se observa que los universitarios están de acuerdo en esta afirmación en un $42.7 \%$ cuando sus familias se encuentran unidas y solo en un $17 \%$ cuando están separadas (véase Tabla 5). El dato más significativo es el que corresponde a la pregunta 13, en donde se identificó un mayor porcentaje cuando los padres estaban separados (50.9\%), por lo que se puede deducir que, en este caso, el padre critica con mayor frecuencia la forma de ser de su pareja. La diferencia encontrada entre los grupos fue estadísticamente significativa $\left(\mathrm{X}^{2}=65.22, \mathrm{p}<.05\right)$.

Adicionalmente, en el indicador "amor", las preguntas 83 y 84 representan la percepción que tienen los estudiantes en relación con el cariño que pueda haber entre sus padres. Al respecto, cuando viven juntos, los porcentajes son más altos que cuando viven separados (véase Tabla 6). En este caso, al utilizar la $X^{2}$ como prueba estadística para comparar los datos, los resultados fueron significati$\operatorname{vos}\left(\mathrm{X}^{2}=158.25, \mathrm{p}<.05\right)$.
Asimismo, en el indicador "compatibilidad de caracteres", la pregunta que más sobresale es la 69 , “¿Tu papá ha abandonado por más de 24 horas la casa a consecuencia de un disgusto?", en donde el mayor porcentaje se encuentra cuando la familia está separada (63\%), a diferencia de cuando se formula la misma pregunta (70) para la madre (15.8 \%). El mismo patrón de inconformidad se puede apreciar en las otras preguntas en el caso de que los padres se encuentren separados, es decir, no hay compatibilidad de caracteres entre los padres (véase Tabla 7). En este caso, también se obtuvo una diferencia estadísticamente significativa $\left(\mathrm{X}^{2}=41.71, \mathrm{p}<.05\right)$.

Y, por último, en la pregunta 61 , “Los momentos difíciles suelen resolverlos juntos?", del indicador "comunicación", los datos muestran porcentajes similares cuando las familias se encuentran unidas $(84.6 \%)$ o cuando están separadas $(80 \%)$. Puede decirse entonces que cuando las familias viven juntas los universitarios perciben que existe mayor comunicación entre la pareja, pues los resultados son diferentes a los encontrados en las otras preguntas -donde se aprecian porcentajes menores cuando los padres se encontraban separados- (véase Tabla 8). Sin embargo, al utilizar la prueba estadística $\mathrm{X}^{2}$, se encontraron diferencias significativas $\left(\mathrm{X}^{2}=201.97, \mathrm{p}<.05\right)$.

Tabla 4

Porcentaje de respuestas afirmativas del indicador "odio y agresión" en familias que viven juntas y en familias separadas

\begin{tabular}{lcc}
\hline \multicolumn{1}{c}{ Pregunta } & Juntos & Separados \\
\hline 26. ¿Tu papá ha agredido físicamente a su pareja? & 14.7 & 39.4 \\
27. ¿Tu mamá ha agredido físicamente a su pareja? & 9.4 & 15.8 \\
46. ¿Tu papá piensa con frecuencia que su pareja la odia? & 12 & 38.2 \\
47. ¿Tu mamá piensa con frecuencia que su pareja la odia? & 9.4 & 21.2 \\
48. ¿Tu papá ha golpeado a su pareja? & 13.4 & 38.8 \\
49. ¿Tu mamá ha golpeado a su pareja? & 8.4 & 12.7 \\
\hline Total & & $27.68 \%$ \\
\hline
\end{tabular}

Nota. $\mathrm{X}^{2}=58.79, \mathrm{p}<.05$.

Tabla 5

Porcentaje de respuestas afirmativas del indicador "concordancia de ideologías" en familias que viven juntas y en familias separadas

\begin{tabular}{lcc}
\hline \multicolumn{1}{c}{ Pregunta } & Juntos & Separados \\
\hline 13. ¿Tu papá critica con frecuencia la forma de ser de su pareja? & 33 & 50.9 \\
14. ¿Tu mamá crítica con frecuencia la forma de ser de su pareja? & 42.1 & 46.7 \\
33. ¿Considera tu papá que tu mamá es un ejemplo para seguir? & 81.7 & 53.3 \\
34. ¿Considera tu mamá que tu papá es un ejemplo para seguir? & 73.8 & 63.3 \\
54. ¿Tus padres tienen formas de pensar muy parecidas? & 42.7 & 17 \\
88. ¿Tu papá depende totalmente de tu mamá? & 87.7 & 13.9 \\
89. ¿Tu mamá depende totalmente de tu papá? & 80.1 & 10.9 \\
\hline Total & $63.01 \%$ & $36.57 \%$ \\
\hline
\end{tabular}

Nota. $\mathrm{X}^{2}=65.22, \mathrm{p}<.05$. 
Tabla 6

Porcentaje de respuestas afirmativas del indicador "amor" en familias que viven juntas y separadas

\begin{tabular}{lcc}
\hline \multicolumn{1}{c}{ Pregunta } & Juntos & Separados \\
\hline 73. ¿Tu papá admira a tu mamá? & 75.1 & 38.2 \\
74. ¿Tu mamá admira a tu papá? & 70.7 & 28.5 \\
83. ¿Piensa tu papá que tu mamá lo quiere? & 83.2 & 23.6 \\
84. ¿Piensa tu mamá que tu papá la quiere? & 81.2 & 24.8 \\
126. ¿Tu papá se siente separado sentimentalmente de su pareja, aunque viven juntos? & 24.6 & 48.5 \\
127. ¿Tu mamá se siente separada sentimentalmente de su pareja, aunque viven juntos? & 27.5 & 50.3 \\
\hline Total & $60.38 \%$ & $35.65 \%$ \\
\hline
\end{tabular}

Nota. $\mathrm{X}^{2}=158.25, \mathrm{p}<.05$.

Tabla 7

Porcentaje de respuestas afirmativas del indicador "compatibilidad de caracteres" en familias que viven juntas y en familias separadas

\begin{tabular}{lcc}
\hline \multicolumn{1}{c}{ Pregunta } & Juntos & Separados \\
\hline 40. ¿A tu papá generalmente le disgusta el comportamiento de su pareja? & 30.4 & 52.1 \\
41. ¿A tu mamá generalmente le disgusta el comportamiento de su pareja? & 38.7 & 49.7 \\
50. ¿Tu mamá y tu papá discuten con mucha frecuencia? & 31.7 & 37.6 \\
69. ¿Tu papá ha abandonado por más de 24 horas la casa a consecuencia de un disgusto? & 16.5 & 63 \\
70. ¿Tu mamá ha abandonado por más de 24 horas la casa a consecuencia de un disgusto? & 10.5 & 15.8 \\
90. ¿Cuándo discuten frecuentemente alguno pierde por completo el control? & 28.3 & 36.4 \\
\hline Total & $26.01 \%$ & $42.43 \%$ \\
\hline
\end{tabular}

Nota. $\mathrm{X}^{2}=41.71, \mathrm{p}<.05$.

Tabla 8

Porcentaje de respuestas afirmativas del indicador "comunicación" en familias que viven juntas y en familias separadas

\begin{tabular}{lcc}
\hline \multicolumn{1}{c}{ Pregunta } & Juntos & Separados \\
\hline 55. ¿La mayoría de las decisiones en casa las toman tu papá considerando a tu mamá? & 73.8 & 21.8 \\
56. ¿La mayoría de las decisiones en casa las toman tu mamá considerando a tu papá? & 78.8 & 24.2 \\
61. ¿Los momentos difíciles suelen resolverlos juntos? & 84.6 & 80 \\
108. ¿Cree tu papá que después de los años de vivir juntos conoce a tu mamá? & 80.9 & 33.3 \\
109. ¿Cree tu mamá que después de los años de vivir juntos conoce a tu papá? & 84.6 & 37 \\
\hline Total & $80.54 \%$ & $39.26 \%$ \\
\hline
\end{tabular}

Nota. $\mathrm{X}^{2}=201.97, \mathrm{p}<.05$.

\section{DISCUSIÓN}

Con respecto a las relaciones de pareja, Soto (2015) señala que es importante tomar en consideración aspectos como la satisfacción sexual, la compatibilidad de caracteres, las aspiraciones y metas comunes, la concordancia de ideologías, la afinidad de valores y la coincidencia en el proyecto de vida para alcanzar la estabilidad; aspectos que tienen que ver con las áreas de la vida cotidiana relacionadas con la educación, valores, hábitos, estilos de vida, ideologías, nacionalidades, lenguas, trabajo, higiene, alimentación y ocio. Asimismo, sobresale el hecho de que la percepción de satisfacción es un factor importante para la consolidación de las relaciones de pareja; percepción relacionada con áreas como lo cotidiano (roles, labores domésticas, rituales, ocio, acuerdos sobre la generación y distribución de recursos), lo emocional (la nutrición relacional, compatibilidad de caracteres, reconocimiento, valoración y apoyo) y la cognición (creencias y valores) (Soto, 2015).

En México, el número de divorcios - una separación conyugal- ha ido en aumento. Por ejemplo, según el INEGI (2017), en 2015 se registraron 123883 casos, un incremento del $136.4 \%$ con respecto al año 2000 , siendo las principales causas el consentimiento mutuo $(50.8 \%)$ y la separación voluntaria unilateral (34.4\%). Esto permite considerar que una buena proporción de los jóvenes vive en un ambiente familiar en donde los padres se encuentran separados, aspecto que no debe pasar desapercibido.

En los resultados de este estudio los datos muestran que existieron diferencias significativas entre la percepción que tienen los universitarios hacia la formación de pareja 
de sus padres cuando viven juntos o cuando se encuentran viviendo separados. Específicamente, se encontró que los estudiantes que viven en una familia en donde los padres son unidos perciben que en la relación de pareja de sus padres hay mayores niveles de comunicación. Dichos resultados son similares a los señalados por Garcés y Palacio (2010), en donde se identificó que el grado de comunicación es mejor en familias que viven juntas, a diferencia de aquellas en donde el estudiante vive solamente con uno de sus progenitores.

En cuanto a las aspiraciones compartidas, manifestaciones amorosas, compatibilidad de caracteres y pocas expresiones agresivas, los resultados son similares a los encontrados en otros estudios como factores clave sobre la elección de pareja y la formación de la familia (García, 2010; Garrido et al. 2008; Padilla \& Díaz-Loving, 2013).

En el caso de los universitarios que viven en familias separadas, los resultados reflejan que los estudiantes perciben la relación de pareja de sus padres con mayores manifestaciones de odio. Específicamente, en este indicador se evidenció que los padres (hombres) fueron los protagonistas de situaciones conflictivas que generaron agresión dentro de la familia, dato que concuerda con lo reportado por Garcés y Palacio (2010) con respecto a que los episodios de maltrato físico fueron mucho mayores en los padres.

Un hallazgo que resulta contrastante es el encontrado en el indicador "compatibilidad de caracteres", en donde los datos revelan que los universitarios perciben mayor compatibilidad de caracteres en sus padres cuando se encuentran separados que cuando están juntos. Una posible razón sería que, al haber la necesidad de llegar a acuerdos sobre los hijos, existe mayor disponibilidad para tratar asuntos familiares que puedan llegar a ser compartidos, entendidos y aceptados por ambos padres para fortalecer la elección previa de la pareja y la permanencia con ella (Soto, 2015).

Como conclusión, puede resaltarse que existen diferencias en la percepción que tienen los universitarios sobre la relación de pareja de sus padres cuando viven juntos y cuando viven separados; que los indicadores que reflejaron estabilidad en la pareja fueron "aspiraciones y metas comunes", "concordancia de ideologías", "amor" y "comunicación”; que los indicadores que reflejaron inestabilidad en la pareja fueron "odio y agresión" y "compatibilidad de caracteres"; y que la percepción que tengan los jóvenes sobre la relación de pareja de sus padres podría influir en la elección futura de su pareja y la formación de su familia.

Por último, resulta oportuno mencionar que este estudio abre las puertas para investigaciones futuras acerca de la influencia que puede tener la percepción de los jóvenes con respecto a las relaciones de pareja de sus padres en sus relaciones afectivas futuras y la forma en que esta puede influir en aspectos como el desempeño académico, la autoestima, el proyecto de vida, el desarrollo laboral, la relación de pareja, la depresión, la ansiedad o las adicciones, entre otros.

\section{REFERENCIAS}

Arch. M. (2010). Divorcio conflicto y consecuencias en los hijos: implicaciones para recomendaciones de guarda y custodia. Papeles del Psicólogo, 3(2), 183-190

Ato, M., \& Vallejo, G. (2015). Diseños de Investigación en Psicología. Madrid, España: Ediciones Pirámide.

De la Torre, J. (2005). Las relaciones entre padres e hijos después de las separaciones conflictivas. Apuntes de Psicología, 23(1), 101-112. Recuperado de http://www.apuntesdepsicologia.es/index.php/revista/article/view/83

Durán, A., Medina, A., González, N., \& Rolón, I. (2007). Relación entre la experiencia de la separación parental y la construcción de un proyecta de vida del joven y la joven universitarios. Universitas Psychologica, 6(3), 713-725. Recuperado de http://www.redalyc.org/pdf/647/64760320.pdf

Estrada, L. (1993). El ciclo vital de la familia. México: Posada.

Fontana, M., \& Fernández, M. (2011). Evaluación de la satisfacción conyugal e interacción padres-hijos en familias de la comunidad de Madrid. International Journal of Developmental and Educational Psychology, 1(2). 511-520. Recuperado de http://infad.eu/RevistaINFAD/2011/n1/ volumen2/INFAD_010223_511-520.pdf

Garcés, M., \& Palacio, J. E. (2010). La comunicación familiar en asentamientos subnormales de Montería. Psicología desde el Caribe, 25, 1-29. Recuperado de http://www.redalyc. org/articulo.oa? $\mathrm{id}=21315106002$

García, M. (2010). Construcción y validación de un instrumento de elección de pareja en las áreas de interacción y características de la pareja. En S. Rivera, R. Díaz-Loving, I. Reyes, R. Sánchez \& L. Cruz, La Psicología Social en México (pp. 97-128). México: AMEPSO, UNAM.

García, M., \& Guzmán, R. (2010). De la elección a la disolución de la relación de pareja: atracción, valores y estatus. En S. Rivera, R. Díaz-Loving, I. Reyes, R. Sánchez \& L. Cruz. La Psicología Social en México (pp. 199-207). México: AMEPSO, UNAM.

García, M., \& Martínez, J. (2010). Construcción y validación de un instrumento de elección de pareja en las áreas de interacción y características de la pareja. Medición en Psicología, 97-128. Recuperado de http://www.redalyc.org/ pdf/292/29210109.pdf

García, M., Rivera-Aragón, S., Diaz-Loving, R., \& Reyes-Lagunes, I. (2015). Continuidad y cambio en la familia. Factores intervinientes. México: Manual Moderno. 
Garrido, A., Reyes, A., Torres, L., \& Ortega, P. (2008). Importancia de las expectativas de pareja en la dinámica familiar. Enseñanza e Investigación en Psicología, 13(2), 231-238. Recuperado de http://www.redalyc.org/ pdf/292/29213203.pdf

INEGI (2017). Estadísticas a propósito del.... 14 de febrero, matrimonios y divorcios en México. Instituto Nacional de Estadística y Geografía. México. Recuperado de http:// www.inegi.org.mx/sistemas/olap/Proyectos/bd/continuas/nupcialidad/Divorcios.asp?s=est\#

Lemes, A. (2016). Mejorando nuestra relación. Programa de intervención para familias con progenitores separados. Universidad de la Laguna, Tenerife, España.

O’Donohue, W., Beitz, K., \& Cummings, N. (2008). A model for constructs relevant to child custody evaluations. Journal of Forensic Psychology Practice, 7(4), 125-139.Recuperado de http://www.tandfonline.com/doi/pdf/10.1300/ J158v07n04_05?needAccess $=$ true

Oudhof, H., \& Robles, E. (2014). Familia y crianza en México. México: Fontamara

Padilla, N., \& Díaz-Loving, R. (2013). Premisas familiares y socioculturales del emparejamiento. Enseñanza e Investigación en Psicología, 18(2), 249-262. Recuperado de http:// www.redalyc.org/articulo.oa?id=29228336003

Silva, S., \& Ortiz, L. (2014). Preferencias en la elección de pareja entre estudiantes universitarios: un análisis según sexo. Tendencias en Psicología, 1(1), 16-27.http://revistas.upagu.edu.pe/index.php/TP/article/view/322/247
Soto, F. R. (2015). Factores que intervienen en la elección de pareja de jóvenes mexicanos. REDES, 32, 71-83. Recuperado de http://redesdigital.com.mx/index.php/redes/article/ view $/ 58$

Torres, L., Ortega, P., Garrido, A., \& Reyes, A. (2008). Dinámica familiar en familias con hijos e hijas. Revista Intercontinental de Psicología y Educación, 10(2), 31-56. Recuperado de http://www.redalyc.org/articulo.oa?id=80212387003

Torres, L., Reyes, A, Ortega, P., \& Garrido, A (2015). Dinámica familiar: formación de identidad e integración sociocultural. Enseñanza e Investigación en Psicología, 20(1), 48-55.

Valdés, A., \& Aguilar, J. (2012). Desempeño académico en hijos de padres casados y divorciados. Revista Mexicana de Orientación Educativa, VIII(20), 24-32.

Vallejo, R., Sánchez-Barranco, F., \& Sánchez-Barranco, P. (2004). Separación o divorcio: trastornos psicológicos en los padres y los hijos. Revista de la Asociación Española de Neuropsiquiatría, 92, 91-110. Recuperado de http://www. redalyc. .org/articulo.oa? $\mathrm{id}=265019658006$

Vásquez, G., Sánchez, T., Navarro, L., Romero, E., Pérez, C., \& Kumazawa, I. (2003). Instrumento de medición de la dinámica de la familia nuclear mexicana: un enfoque cuantitativo. Boletín Médico del Hospital Infantil Mexicano, 60, 33-52. Recuperado de http://www.imbiomed.com.mx/1/1/articulos.php?method=showDetail\&id_articulo=10837\&id_ seccion $=981 \&$ id_ejemplar $=1116 \&$ id_revista $=20$ 